\title{
Sûreté nucléaire et déchets radioactifs $\left({ }^{*}\right)$
}

\author{
A. GEY $(* *)$ \\ (Manuscrit reçu le 23 mars 1979)
}

\section{RÉSUMÉ}

Pour ceux qu'intéresse la sûreté nucléaire, les principes essentiels de cette discipline et ses méthodes d'évaluation du risque sont souvent inconnus. Les lignes suivantes ont été écrites pour les instruire de ce sujet, avec si possible des mots courants et des explications simples, parfois schématiques. Les principes et méthodes de sûreté sont appliqués bien entendu aux installations nucléaires, mais ils le sont aussi aux rejets de ces installations, tels qu'effluents et déchets. Cet article tente de donner une idée de l'importance des déchets radioactifs solides et de leur destinée.

\begin{abstract}
The fundamental principles of nuclear safety and the risk assessment methods are often unknown to people concerned by this field. The following lines have been written to inform them using if possible usual words and simple, sometimes schematic, explanations. Safety principles and methods are applied to nuclear plants, of course; but they are also applied to their radioactive releases and wastes. This article is a tentative to give an idea of the importance of solide radioactive wastes and of their fate.
\end{abstract}

\section{"J'appelle un chat un chat et Rollet un fripon"... Boileau.}

Il est des mots tabous; des mots qu'on hésite à prononcer parce qu'ils semblent faire scandale dans le contexte où ils sont utilisés. Tel est le mot « risque » dans le contexte de l'énergie atomique. Pourtant la vie est confrontée à quantité de risques : risque de monter dans une voiture, risque de franchir le seuil d'une maison, risque de recevoir une météorite sur la tête. Tous les risques n'ont pas même valeur dissuasive, et on dit même qu'on « risque de gagner " en prenant un billet de loterie! Le risque est donc un aléa qui entraîne, ou non, un dégât. Comme tout ce à quoi est conîrontée l'espèce humaine, l'énergie nucléaire présente donc des risques; et, se référant à BoILEAU,

(*) Journée de Médecine préventive et sociale, Dinard, 14 septembre 1978.

(**) Commissariat à l'Énergie atomique, Institut de Protection et de Sûreté nucléaire, Département de Sécurité des Matières nucléaires, B.P. $n^{\circ} 6,92260$ Fontenay-aux-Roses. 
il faut bien le dire et nommer les choses par leur nom. Mais la particularité de l'énergie atomique, par comparaison avec les autres produits de l'industrie humaine, est d'avoir scrupuleusement réduit à un niveau infime la probabilité et l'ampleur des risques qui lui sont inhérents : c'est la raison d'être de la "sûreté nucléaire ".

En effet, celle-ci a pour but de déceler, recenser, évaluer, neutraliser, surveiller et combattre tous les risques potentiels présentés par l'emploi de l'énergie atomique. La sûreté nucléaire s'exerce donc dans la prévention des risques, la surveillance des dispositifs qui s'opposent à la manifestation de ces risques, et les contremesures dans le cas où, malgré tout, ces dispositifs seraient mis en défaut.

Et ceci dans tous les cas : circonstances normales, bien entendu; circonstances transitoires inhérentes au fonctionnement; circonstances accidentelles enfin.

Pour illustrer ces considérations quelque peu abstraites, on se référera à l'exemple le plus démonstratif : celui des réacteurs (et même plus particulièrement à celui des réacteurs à eau sous pression). Étant bien entendu que cet exemple peut sans grande peine se généraliser à d'autres types d'installations (ou d'activités nucléaires) : usines, transports, stockages, etc.

\section{L'OBJET : LE RÉACTEUR NUCLÉAIRE}

Il n'est peut-être pas inutile de rappeler que la fission est un phénomène nucléaire au cours duquel le noyau fissile est brisé, émet de l'énergie, et libère en moyenne 2 à 3 neutrons.

Les fragments de fission sont radioactifs. Les neutrons engendrés peuvent provoquer à leur tour des fissions, développant ainsi le processus connu sous le nom de réaction en chaîne. Si la chaîne est divergente, on peut aboutir à une explosion; si elle est convergente, la chaîne s'éteint plus ou moins rapidement. Un réacteur nucléaire est un assemblage de matière fissile dans lequel le processus de réaction en chaîne n'est ni convergent, ni divergent; on dit que le système est à l'état critique. Dans un tel état, à toute fission correspond un seul neutron utile parmi les deux ou trois libérés; les autres ont été capturés ou se sont enfuis. Un réacteur nucléaire produit donc de l'énergie - c'est sa raison d'être - des neutrons en quantité quasi-constante, des rayonnements électromagnétiques, et des produits de fission. Tout ceci se passe dans le cæuur du réacteur; ce cœur est composé d'éléments combustibles gainés, plongés dans un fluide qui peut être modérateur, caloporteur, ou les deux. Dans le cas des réacteurs à eau sous pression, qui va servir ici d'exemple, l'eau est à la fois modératrice et caloporteuse. Le réacteur ressemble donc à un énorme autocuiseur dans lequel le cœur joue la fonction d'un thermo-plongeur.

Cette eau est mise en mouvement par des pompes, sa chaleur échangée dans des générateurs de vapeur (G.V.), et la vapeur ainsi produite actionre les turbines, d'où l'électricité. 
En résumé, le réacteur se compose donc : d'un assemblage combustible dit « cœur »; d'une cuve et d'un circuit " primaire » dans lesquels circule l'eau qui est entrée en contact avec le cœur et de plusieurs générateurs de vapeur (G.V.), dans lesquels circule une eau différente de la précédente, utilisée à produire de l'énergie et réfrigérée de façon indépendante, soit par l'air, soit par une troisième eau.

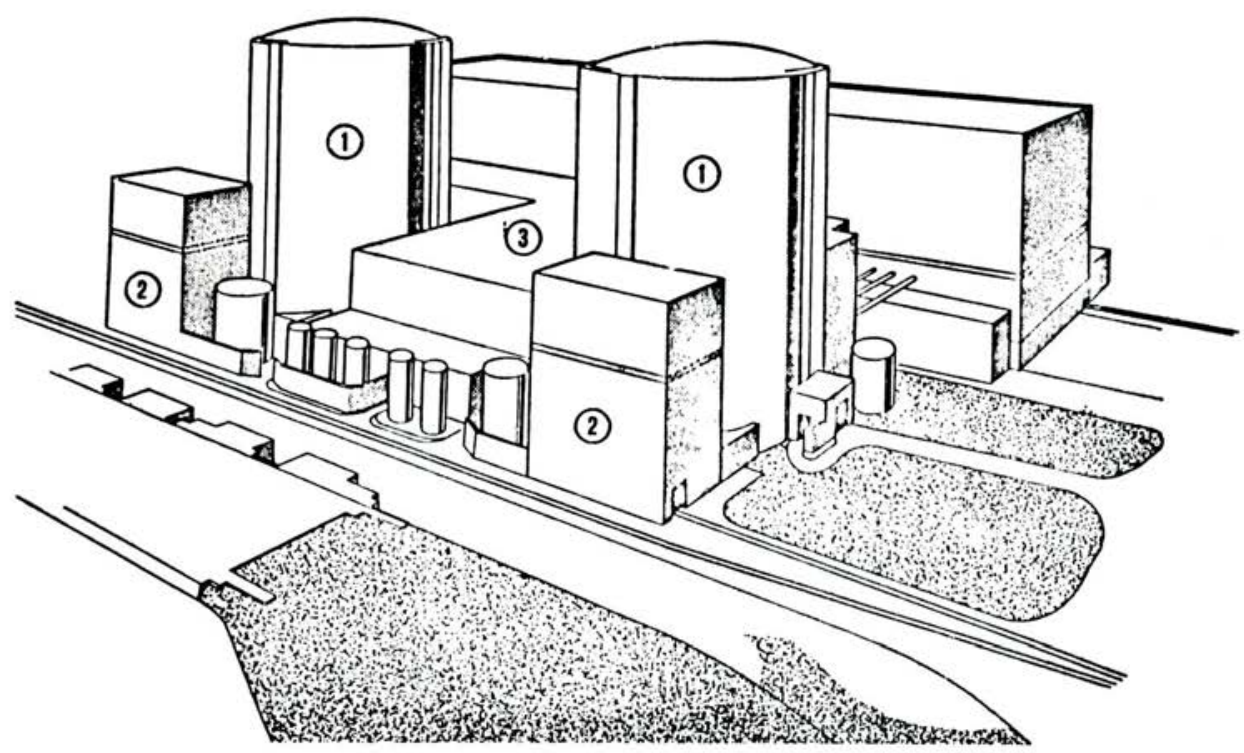

FIG. 1. - Vue d'ensemble d'un réacteur à eau sous pression: 1, bâtiment réacteur; 2, bâtiment combustible; 3, bâtiment des auxiliaires nucléaires (B.A.N.).

Cœur, circuit primaire et G.V. sont placés dans un bâtiment, dit bâtiment réacteur, lui-même entouré d'autres bâtiments nucléaires tels que bâtiment " combustible " ou bâtiment des auxiliaires nucléaires "B.A.N. " ( fig. 1).

La sûreté nucléaire va consister à s'assurer que rien de ce qui se produit à l'intérieur de cet ensemble n'engendre de risques inacceptables, et particulièrement de risques radioactifs, ni pour les travailleurs, ni pour la population.

\section{LA MÉTHODE: LES «BARRIÈRES »}

Pour parvenir à un tel résultat, les méthodes peuvent légèrement différer entre nations; mais elles reviennent toutes sensiblement à contenir la radioactivité à l'intérieur d'enveloppes gigognes. Cette méthode, développée en France sous l'impulsion de J. BourgeOIs, a reçu le nom de méthode des barrières. 
Pour reprendre l'exemple du réacteur à eau sous pression, les barrières sont ici au nombre de trois ( fig. 2) : la gaine du combustible; l'enveloppe du réacteur et l'enceinte de confinement.

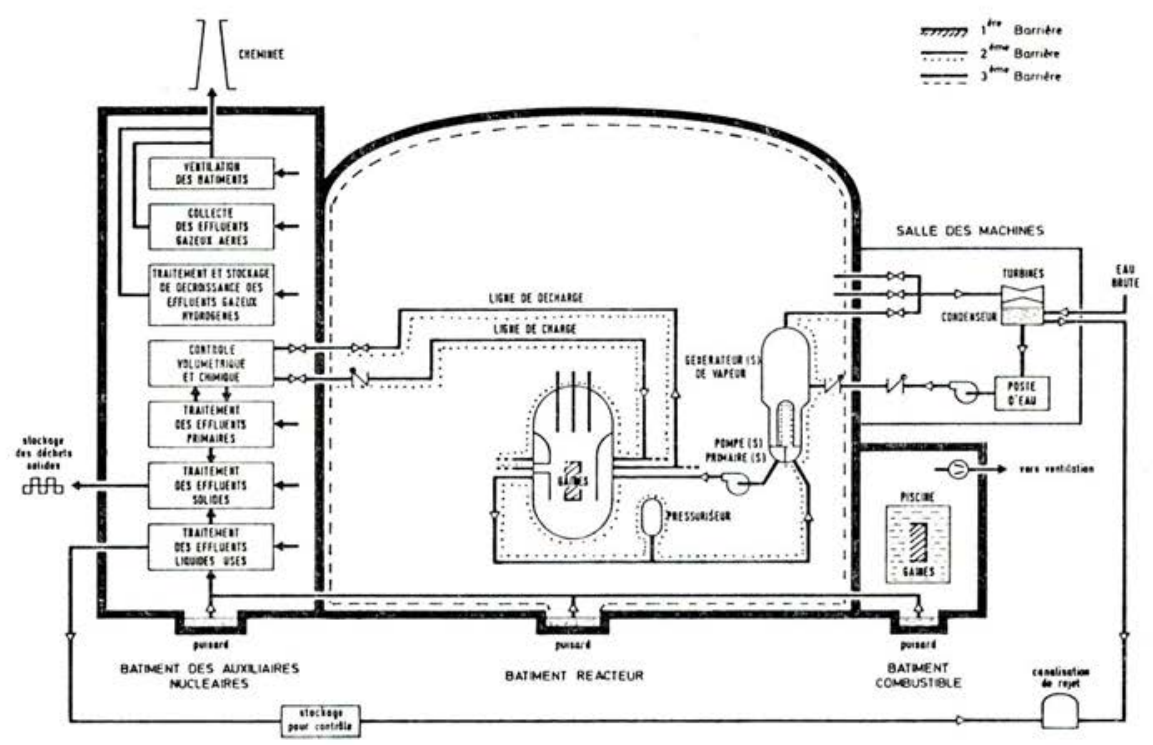

FIG 2 - Représentation schématique des différentes barrières d'une centrale nucléaire à eau sous pression, d'après [4]. Vue cavalière - et partiellement éclaté.

\subsection{LA GAINE.}

La gaine étanche qui enveloppe le combustible évite que les produits de fission (P.F.), gazeux, volatils, ou solides, ne parviennent par migration dans la matière fissile au contact de fluide caloporteur. Cette migration est d'ailleurs plus ou moins rapide selon la forme physique des P.F. : rapide pour les gaz rares (xénons, kryptons) et les iodes, elle l'est déjà moins pour les autres espèces chimiques. Mais si soignée soit-elle, la gaine qui est soumise à d'importantes contraintes thermodynamiques et mécaniques peut parfois se fissurer et donc laisser échapper quelques P.F.; il faut donc interposer une seconde barrière.

\subsection{L'ENVELOPPE.}

Elle constitue la deuxième barrière, composée de tout ce dans quoi circule l'eau primaire : cuve ( fig. 3), circuit primaire, pompes, pressuriseur, partie primaire de l'échangeur dans les G.V., etc. L'eau véhiculée dans cette enveloppe peut, comme on l'a vu, être contaminée par les P.F. Mais elle peut 
tout aussi bien être rendue radioactive par les neutrons du cœur. En particulier, elle contient à coup sûr du tritium, soit du fait de l'activation du deutérium de l'eau naturelle, soit par suite de la réaction nucléaire des neutrons sur l'acide borique techniquement nécessaire à la régulation du réacteur, soit enfin parce que le tritium est un des P.F. échappés des gaines.

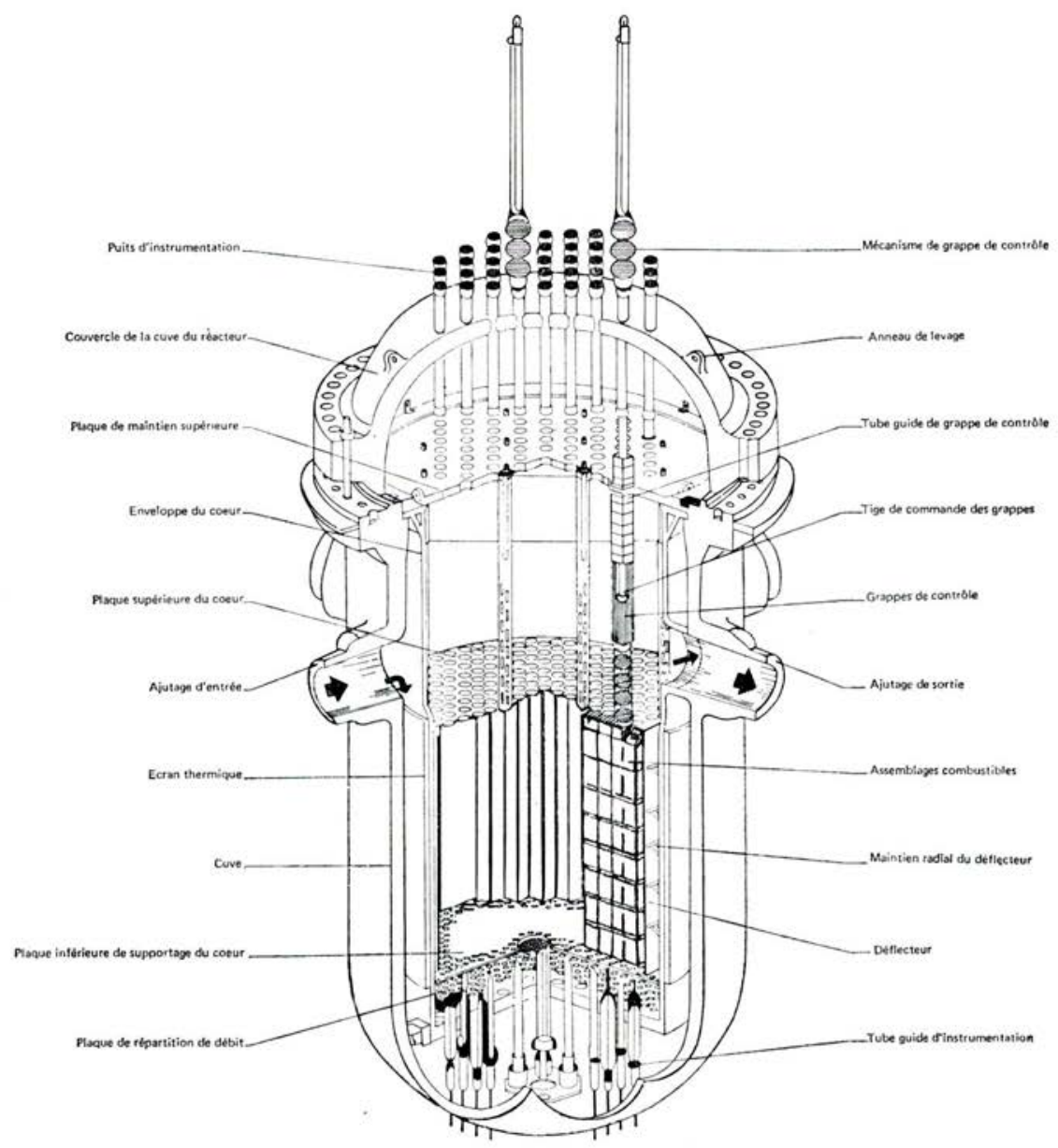

FIG. 3. - Cuve d'un réacteur et structures internes.

L'eau véhicule également des produits de corrosion activés : manganèse, fer, cobalt, etc. Une très grande partie de la sûreté portera donc sur l'intégrité de cette deuxième barrière; pour le cas très probable où cette intégrité serait mise en défaut, on interpose une troisième barrière.

voL. $14-\mathrm{N}^{\circ} 3$ 


\subsection{L'ENCEINTE DE CONFINEMENT ( fig. 4) :}

A partir de cette troisième barrière; l'espèce humaine apparaît : les travailleurs à l'intérieur de l'installation, la population, à l'extérieur. Si tout le nécessaire est fait pour les protéger, il n'empêche que cette troisième et

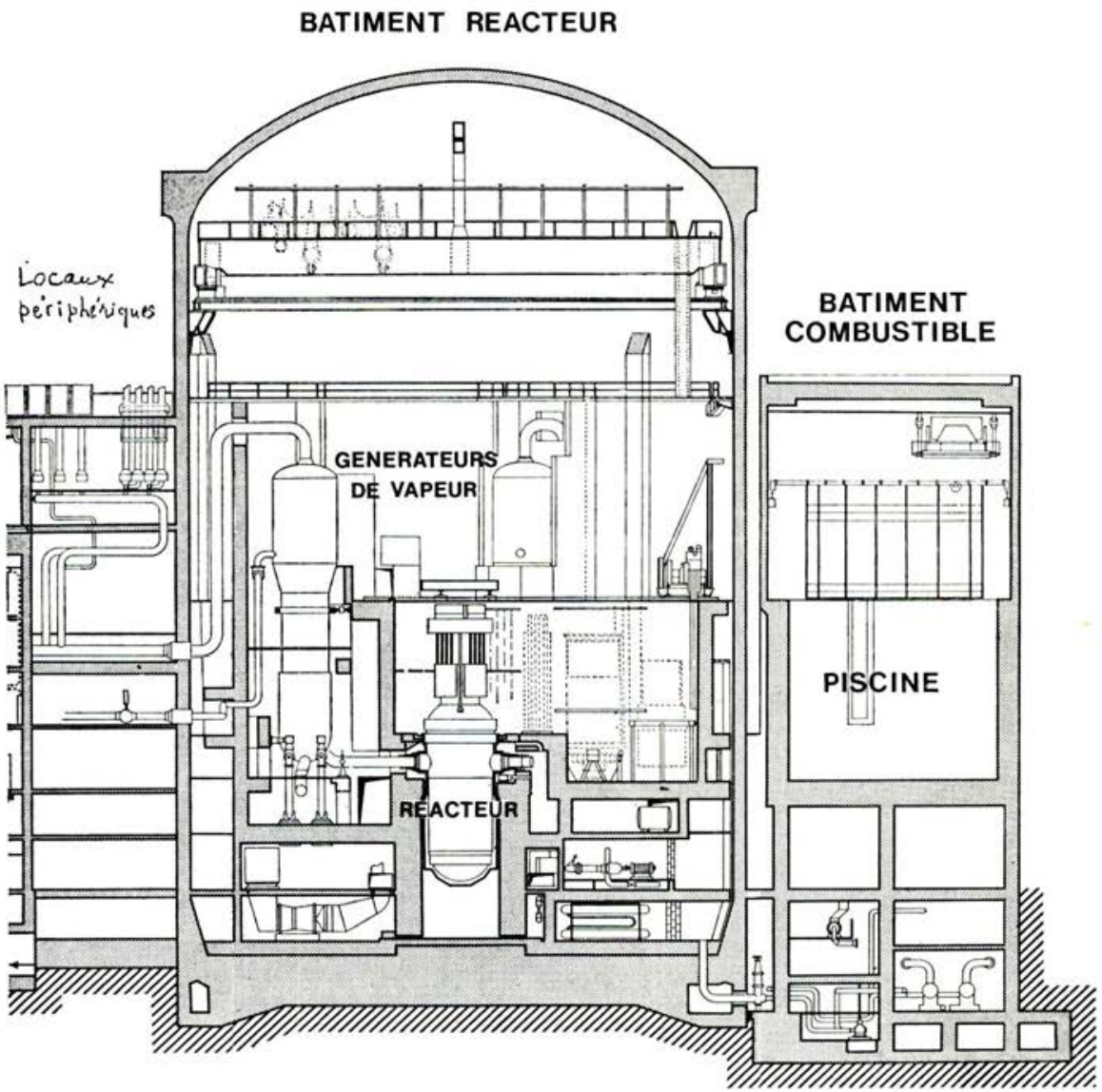

FIG. 4. - Exemple d'enceintes de confinement : bâtiment réacteur et bâtiment combustible.

dernière barrière est le dernier recours pour la protection de l'environnement. Aussi doit-elle être particulièrement robuste, et le confinement qu'elle assure particulièrement soigné.

Cette enceinte peut être en superstructure comme c'est le cas le plus courant. Elle est protégée contre les projectiles internes par des écrans; elle doit pouvoir résister aux projectiles externes, par exemple aux fragments 


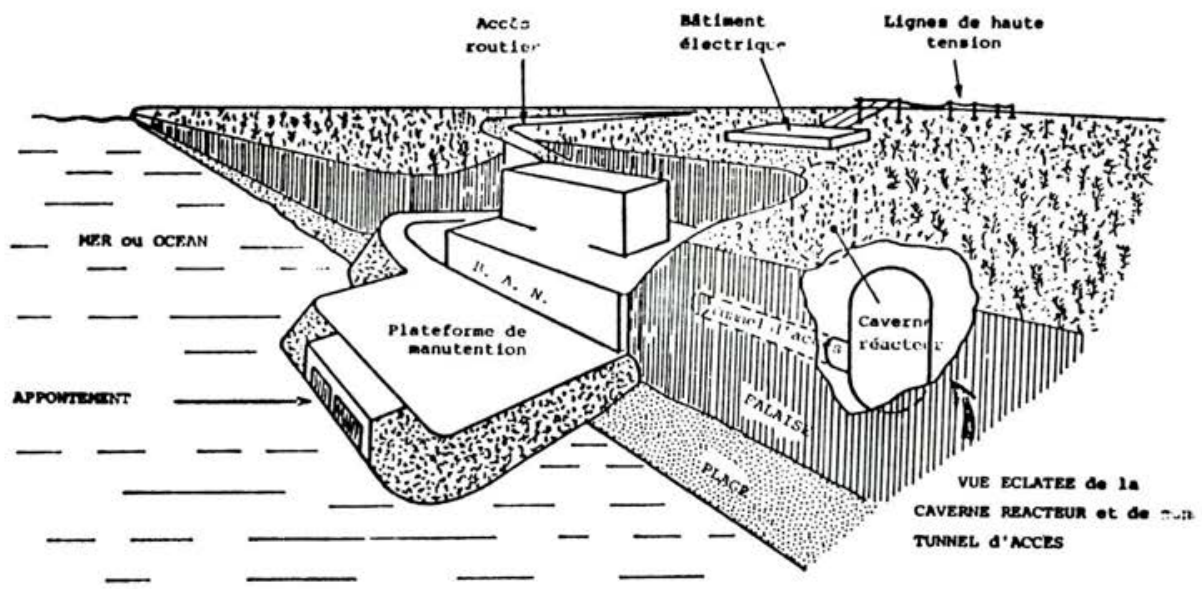

FIG. 5. - Architecture enterrée (en tunnel) Vue cavalière - et partiellement éclaté.
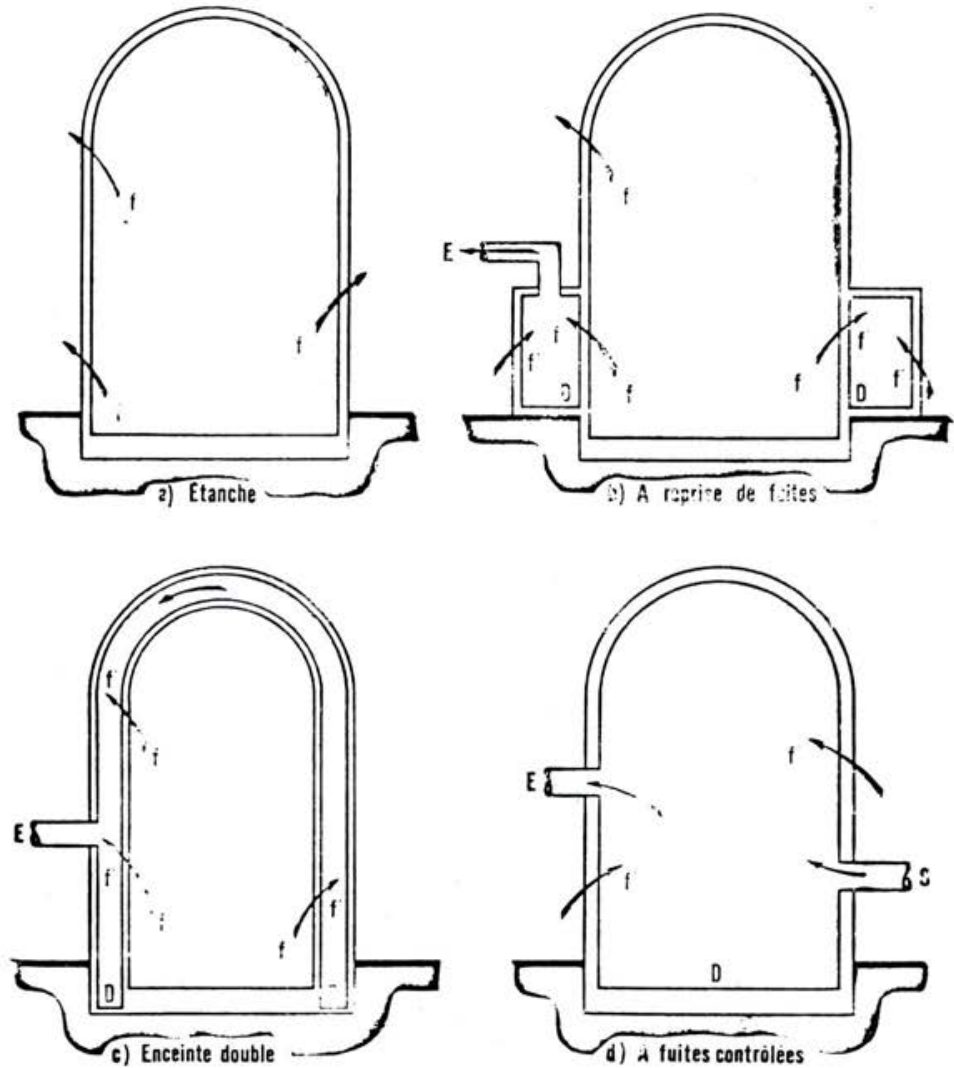

FIG. 6. - Nomenclature des enceintes : étanchéité.

$\mathrm{f}, \mathrm{f}^{\prime}$ : fuites et infiltrations; $\mathrm{S}$ : soufflage protégé; $\mathrm{E}:$ extraction filtrée; $\mathrm{D}:$ dépression 


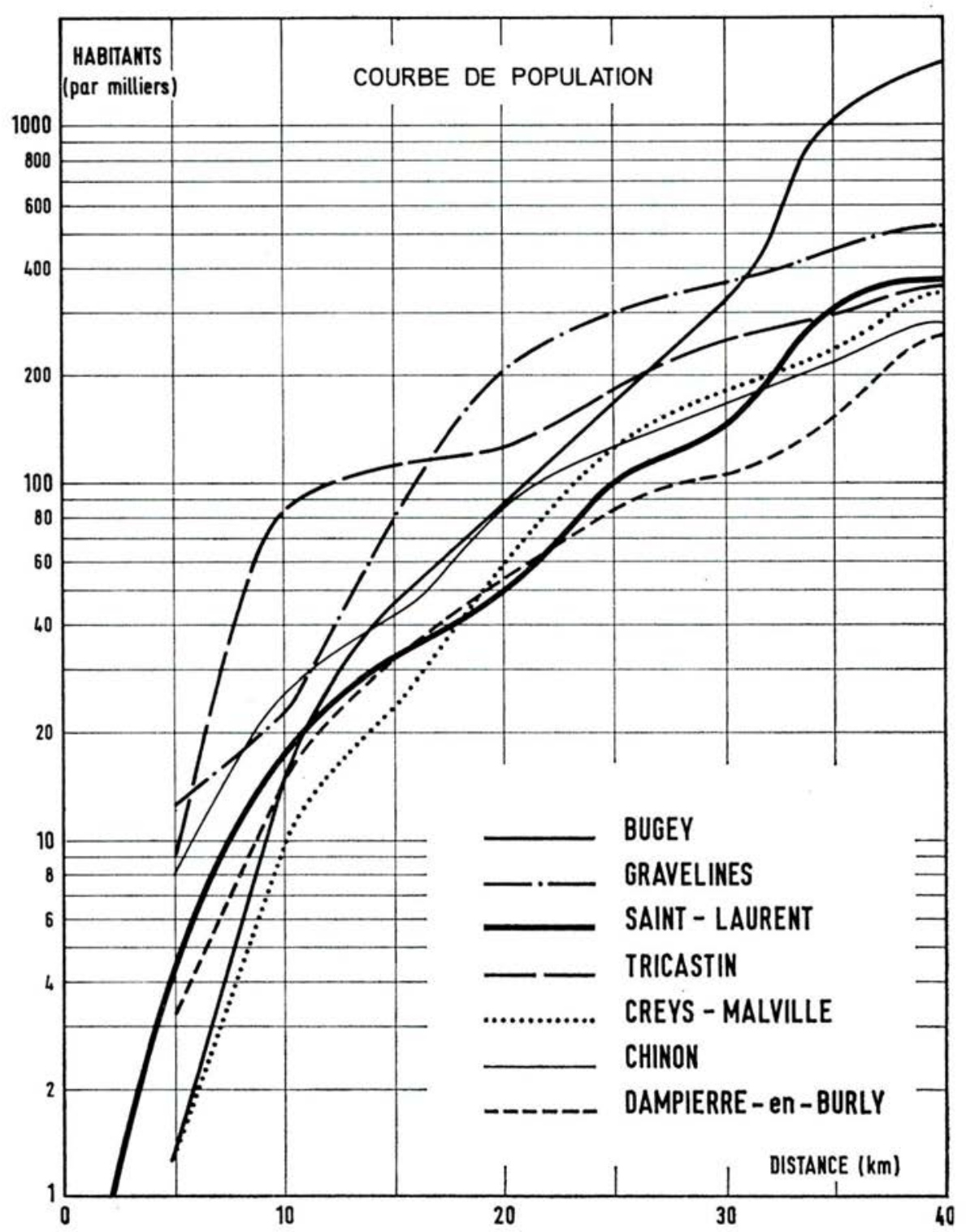

FIG. 7. - Courbes de répartition de la population pour quelques sites en exploitation ou prévus, d'après [19]. 
de volants d'alternateurs ruptés. Mais elle peut également être semi-enterrée, ou enterrée, ce qui accroît son invulnérabilité ( fig. 5).

En ce qui concerne le confinement, celui-ci peut être assuré de diverses manières; on aboutit ainsi à des enceintes dites étanches, à reprises de fuites doubles, à fuites contrôlées ( $f i g$. 6). Une peau d'étanchéité en acier peut parfois venir garnir le béton à l'intérieur. Les traversées indipensables pour l'alimentation électrique, le passage des fluides, l'évacuation des rejets, l'introduction du combustible, les va-et-vient de personnel sont particulièrement soignés.

\subsection{L'environnement. Choix des sites}

De ce qui précède, on déduit qu'il n'est donc pas indifférent de choisir un site nucléaire selon les agressions qu'il peut faire subir à l'installation : crues, raz-de-marée, explosions d'hydrocarbures, séismes, etc. et les agressions que l'installation pourrait faire subir au site par ses rejets, que ceux-ci soient concertés ou non. En particulier, les rejets atmosphériques font l'objet d'un examen particulièrement sévère.

Le premier objectif de la sûreté étant de prévoir les détriments possibles, cette prévision se fondera entre autres choses, sur les courbes de répartition des populations autour des points de rejets ( fig. 7). On voit, à ce propos, combien le problème peut se présenter de façon différente selon qu'on se trouve en France, en Belgique ou aux États-Unis.

Voici donc passées en revue les barrières qui concrétisent les obstacles opposés à la propagation indésirable de la radioactivité. Mais quelle confiance leur accorder? C'est le but de l'analyse de sûreté; son outil privilégié est la méthode probabiliste.

\section{L'ÉVALUATION DE LA SURETÉ: ARBRES D'ÉVÉNEMENTS, ARBRES DE DÉFAILLANCES}

Si bien étudiées que soient les barrières, et correctement prévues les agressions, la technologie des installations nucléaires - et des réacteurs entre autres - peut néanmoins être mise en défaut. C'est d'ailleurs le propre de toute entreprise humaine. Aussi l'efficacité des barrières est-elle surveillée, et, si elle venait à descendre au-dessous des limites tolérables, des contremesures seraient-elles prises pour rétablir le niveau de sûreté requis. Ces actions de sécurité peuvent également subir des défaillances, individuellement ou à la suite d'une cascade d'événements. L'évaluation de la sûreté consiste donc à apprécier les conséquences des incidents et des accidents susceptibles de provoquer une brèche dans les barrières précédemment évoquées. La méthode dite des arbres (arbres d'événements, arbres de défaillances) permet de quantifier le risque et donc de l'apprécier objectivement. 


\subsection{ARBRES D'ÉVÉNEMENTS}

Un exemple d'arbre d'événements peut être donné par l'analyse d'un accident de dépressurisation d'un réacteur à eau sous pression (fig. 8). On voit, qu'à partir de l'accident initial (rupture de la branche froide du

Exemple simplifié d'arbre d'événements dans un accident par défaut de refroidissement.
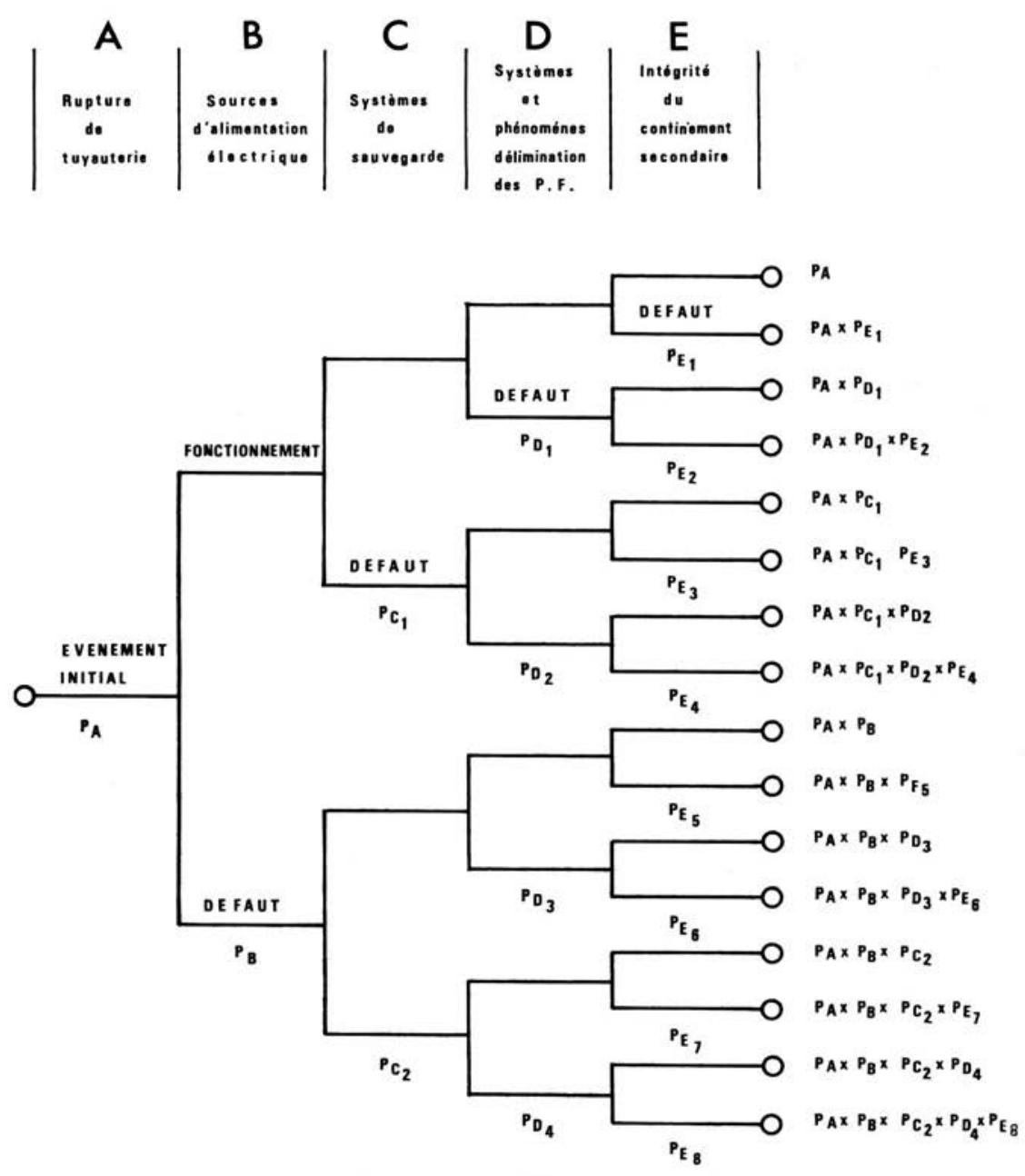

Fig. 8. - Exemple simplifié d'arbre d'événements dans un accident par défaut de refroidissement, schéma de base. 
circuit primaire à l'entrée de la cuve), les situations ou les événements possibles peuvent être classés selon leurs conséquences; ainsi : pas d'électricité, pas de moyens de sauvegarde, etc.

On voit immédiatement que certaines branches n'ont aucun sens; ainsi, il est inutile de poursuivre les investigations au-delà de la cascade : rupture de tuyauterie $E T$ perte d'énergie électrique. L'arbre d'événements se simplifie
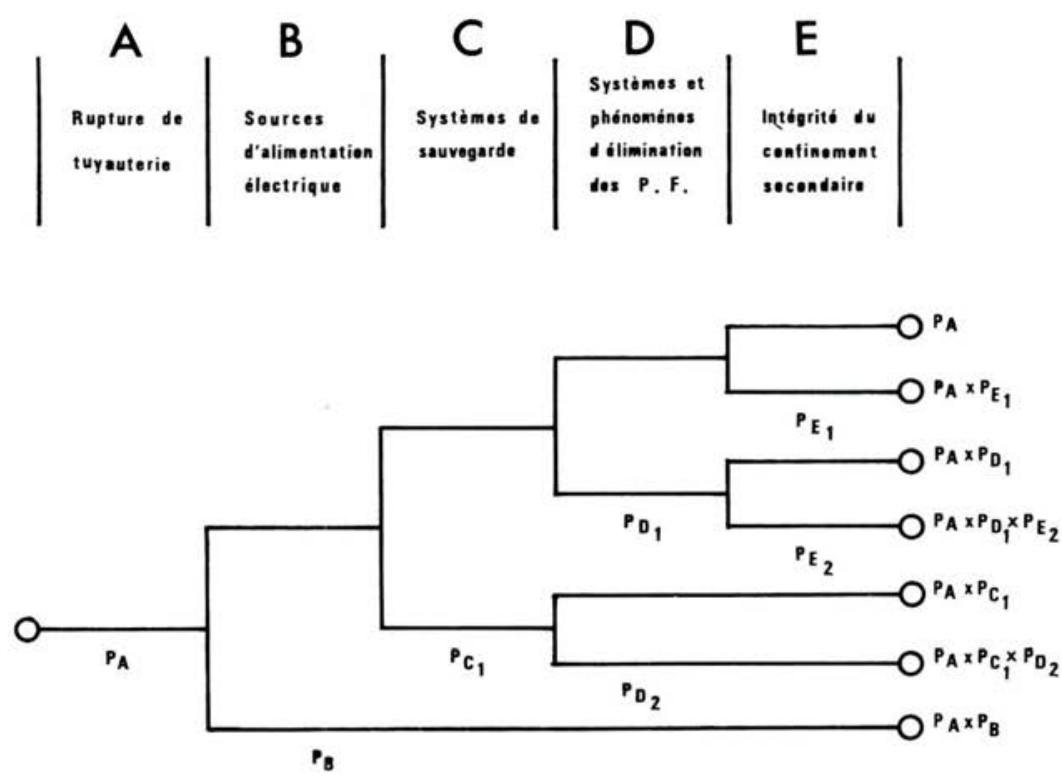

Fig. 9. - Exemple simplifié d'arbre d'événements dans un accident par défaut de refroidissement, schéma réduit (par élimination des séquences illogiques).

Nota : Comme la probabilité de défaut $P$ est beaucoup plus petite que 1 la probabilité de fonctionnement correct $1-P$ est toujours très voisine de 1 . Ainsi la probabilité associée aux branches supérieures est supposée égale à 1 .

aussitôt et on voit que, si tous les systèmes de sauvegarde fonctionnent, la probabilité de la cascade d'événements se réduit pratiquement à celle de l'événement initiateur lui-même ( $f i g$. 9).

Mais si la méthode des arbres d'événements permet d'accéder aux conséquences, elle permet moins facilement la recherche des événements initiateurs; la méthode des arbres de défaillances, elle, le permet.

\subsection{ARbres DE DÉFAillances}

L'exemple suivant montre de quelle façon on peut rechercher les causes qui empêcheraient l'arrêt d'urgence du réacteur ( fig. 10). Le symbole $\overline{\mathrm{AU}}$ signifie PAS d'Arrêt d'Urgence. L'arrêt d'urgence est provoqué par l'intro-

vOL. $14-\mathrm{N}^{\circ} 3$ 

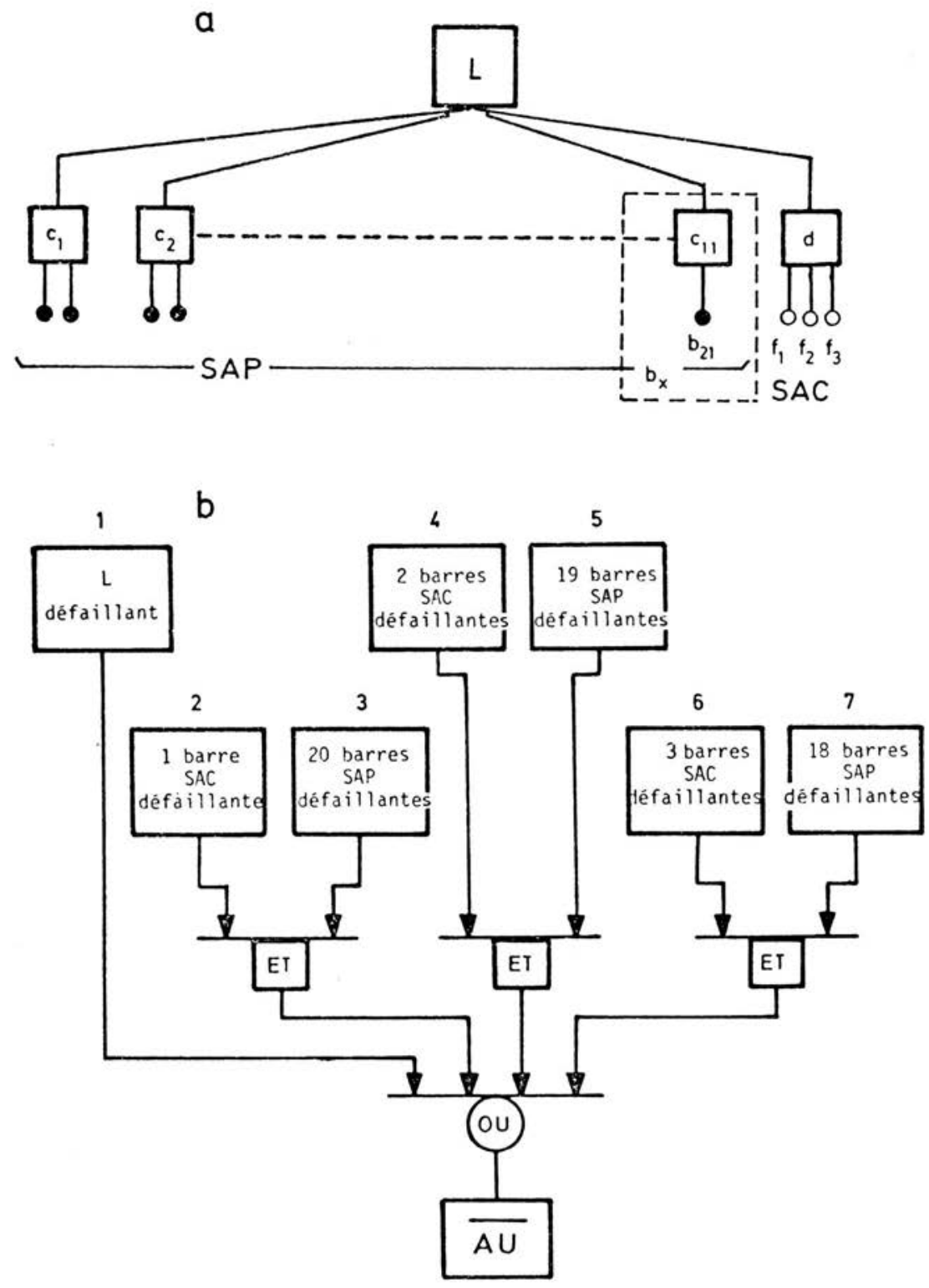

FIG. 10. - Système d'arrêt d'urgence du réacteur, schéma (a) et arbre de défaillances $(b)$, d'après [13]. 
duction dans le réacteur de barres absorbantes de neutrons (barres au bore); l'introduction de ce "poison » neutronique rend le cœur sous-critique; les réactions en chaîne, de divergentes ou stationnaires, deviennent convergentes et le réacteur "s'éteint ".

Les barres de sécurité forment deux groupes : les barres SAP (système d'arrêt principal) et les barres SAC (système d'arrêt complémentaire) plus réactives que les barres SAP. On voit que l'arrêt d'urgence est défaillant dans l'un des cas suivants :

- si le dispositif L, qui élabore le signal de commande de chutes des barres, tombe en panne;

- si l'une des combinaisons 2 et 3 , ou 4 et 5 , ou 6 et 7 des barres est défaillante.

L'examen des arbres de défaillances permet donc de remonter des effets redoutés aux causes multiformes de ceux-ci, et notamment de déceler les modes communs : ce sont des événements, ou des successions d'événements, qui peuvent provoquer des défaillances simultanées sur divers systèmes de sécurité normalement indépendants. Ainsi un séisme imprévisible de très forte intensité pourrait-il à la fois priver le réacteur d'énergie électrique et altérer l'étanchéité de l'enceinte de confinement. Une autre forme de mode commun peut-être décelée dans les défaillances des systèmes qui mettraient en échec plusieurs circuits de sécurité théoriquement indépendants.

Ainsi, la méthode des arbres de défaillances recoupe-t-elle celle des arbres d'événements (ou d'agressions).

Dès lors, on dipose des outils indispensables pour procéder à l'analyse quantitative des risques - donc à l'évaluation de la sûreté.

\section{3. Évaluation de la sûReté}

Partant de tous les événements possibles - incidents, agressions, accidents - on peut alors " monter » les divers "scénarios » possibles avec leurs probabilités d'occurrence (ou risques), et l'ampleur consécutive des dégâts causés (dommages). Selon que le résultat - ou détriment - est acceptable ou non la conception du système ne doit pas, ou doit être revue.

Une telle analyse probabiliste de sûreté a été mise en pratique sur une grande échelle aux États-Unis. La synthèse des résultats a été donnée dans un rapport désormais fameux, le WASH-1400 ou rapport RASMUSSEN.

\subsection{RAPPORT RASMUSSEN}

L'impact particulier de ce rapport qui a évalué les risques (et les détriments) nucléaires, est de les avoir comparés aux risques et aux détriments dits " classiques " par opposition. Bien qu'il ait été contesté, et par des critiques compétents en outre, ces derniers n'ont pu remettre en cause l'essentiel des résultats de la première version du rapport RASMUSSEN.

Les résultats sont éloquents ( fig. 11); aux États-Unis un incendie causant 100 victimes risque de survenir une fois tous les 7 ans; une explosion, une fois tous les 16 ans; une rupture de barrage, une fois tous les 25 ans; et

vOL. $14-\mathrm{N}^{\circ} 3$ 
l'exploitation actuelle de 100 centrales électronucléaires ne causerait un accident faisant plus de 100 victimes q'une fois tous les 10000 ans! ceci, sans tenir compte des progrès qui seront sans aucun doute accomplis en matière de sûreté dans les prochaines décennies.

Ces éléments d'appréciation, pour réconfortants qu'ils soient, ne sont que la condition nécessaire, mais non suffisante, à la construction ou à l'exploitation d'un réacteur nucléaire. Encore faut-il vérifier que ce réacteur

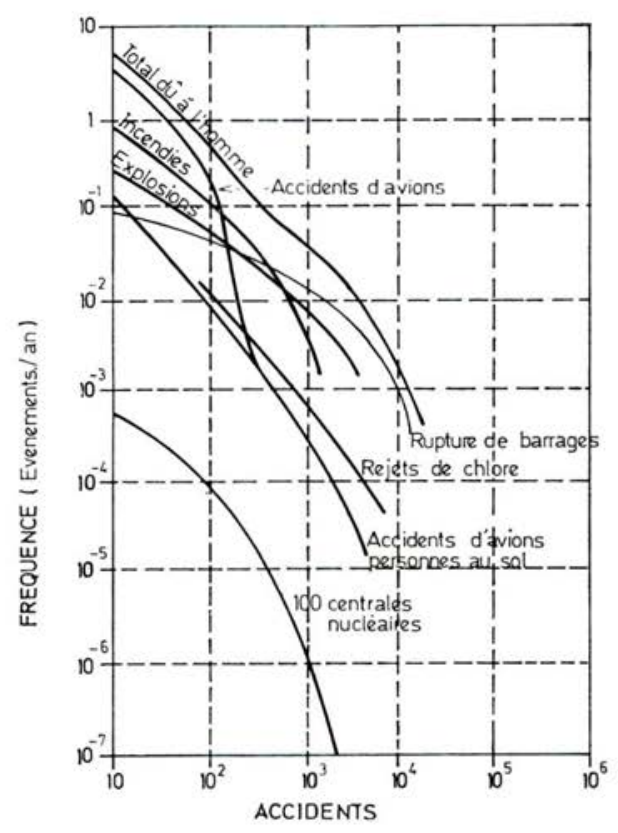

FIG. 11. - Comparaison des probabilités d'accidents dus à des activités humaines (rapport Rasmussen), d'après [18].

est conçu et réalisé en conformité avec les règles en vigueur; chaque installation nucléaire de quelque importance doit donc recevoir l'autorisation des pouvoirs publics, comme la législation française l'exige de tout établissement réputé dangereux, insalubre ou incommode.

\section{LA RÉGLEMENTATION EN MATIÈRE NUCLÉAIRE : PROCÉDURE}

Toute installation nucléaire susceptible de produire ou de manipuler de la radioactivité au-dessus d'un certain seuil, est dite installation nucléaire de base (INB); son responsable est tenu de fournir un rapport de sûreté. 
Ce rapport de sûreté est tout d'abord provisoire : il concerne l'autorisation de construire. C'est une des phases les plus importantes puisque le rapport indique les grands principes sur lesquels sera basée la sûreté nucléaire de l'installation et décrit le site choisi pour l'installer. Le dossier est étudié techniquement par l'organisme conseiller du Ministère de l'Industrie en matière nucléaire (en l'occurrence, le CEA en son Institut de Protection et de Sûreté nucléaire et en particulier le Département de Sûreté nucléaire). Un groupe permanent, composé de membres désignés par arrêté ministériel, donne officiellement son avis au Service central de Sûreté des Installations nucléaires (SCSIN). Celui-ci, après avoir pris l'avis de la Commission interministérielle des Installations nucléaires de Base (CIINB) et recueilli, en particulier, l'avis conforme du Ministère de la Santé publique propose (ou non), au Ministère, le Décret de création de l'INB. Après cette décision qui constitue un tournant capital pour l'installation à venir, un rapport provisoire, puis définitif, suivent une procédure analogue, débouchant successivement sur des autorisations de démarrage puis d'exploitation de l'installation.

Mais, si d'aventure, dans la vie de l'installation, un principe essentiel de sûreté était remis en cause, ou une partie importante de l'installation était rénovée, une nouvelle demande d'autorisation, fondée sur un nouveau rapport (ou partie du rapport), serait exigée.

Du point de vue des rejets dans l'environnement (rejets gazeux, rejets liquides), il existe également des contraintes. Dans le cadre national, les rejets sont soumis à une procédure de déclaration du futur exploitant et d'autorisation de la part des pouvoirs publics. Dans le cadre international, le responsable de l'INB est tenu de se soumettre à une procédure dite de " l'article 37 d'EURATOM ». Le Département de Protection du CEA, Le Service central de Protection contre les Rayonnements ionisants du Ministère de la Santé publique sont, chacun pour leur part, concernés par le respect de ces procédures.

Mais il n'y a pas que des effluents fluides qui soient produits par une installation nucléaire; celle-ci produit aussi des résidus qui, tôt ou tard, prendront une forme solide; ces résidus, inutilisés ou inutilisables, sont des déchets radioactifs; leur destin, transitoire ou final, concerne aussi la sûreté.

\section{LES DÉCHETS RADIOACTIFS}

Les déchets radioactifs sont produits par toutes les installations nucléaires. Mais la majeure partie des déchets aura sans aucun doute pour origine le cycle du combustible dans la production d'énergie nucléo-électrique.

A chaque stade de ce cycle, la production de déchets varie en volume et en nocivité (tableau I). Le volume et la radioactivité des déchets ne sont pas directement représentatifs de leur danger potentiel pour l'homme, danger qui provient éventuellement de l'irradiation externe ou de la contamination interne (par l'air ou par l'eau). Le conditionnement des déchets est tel qu'en général, seul le problème de la contamination de l'eau est à prendre en considération. C'est la raison pour laquelle, dans ce texte, on utilise les termes d'indice de

VOL. $14-\mathrm{N}^{\circ} 3$ 


\section{TABLEAU I}

ORDRE APPROXIMATIF DE GRANDEUR DE L'ENCOMBREMENT IT DE LA RADIOTOXICITÉ RELATIVE DES DÉCHETS SOLIDES ( $\mathrm{GW}_{e}$.an d'énergie nucléo-électrique produite)

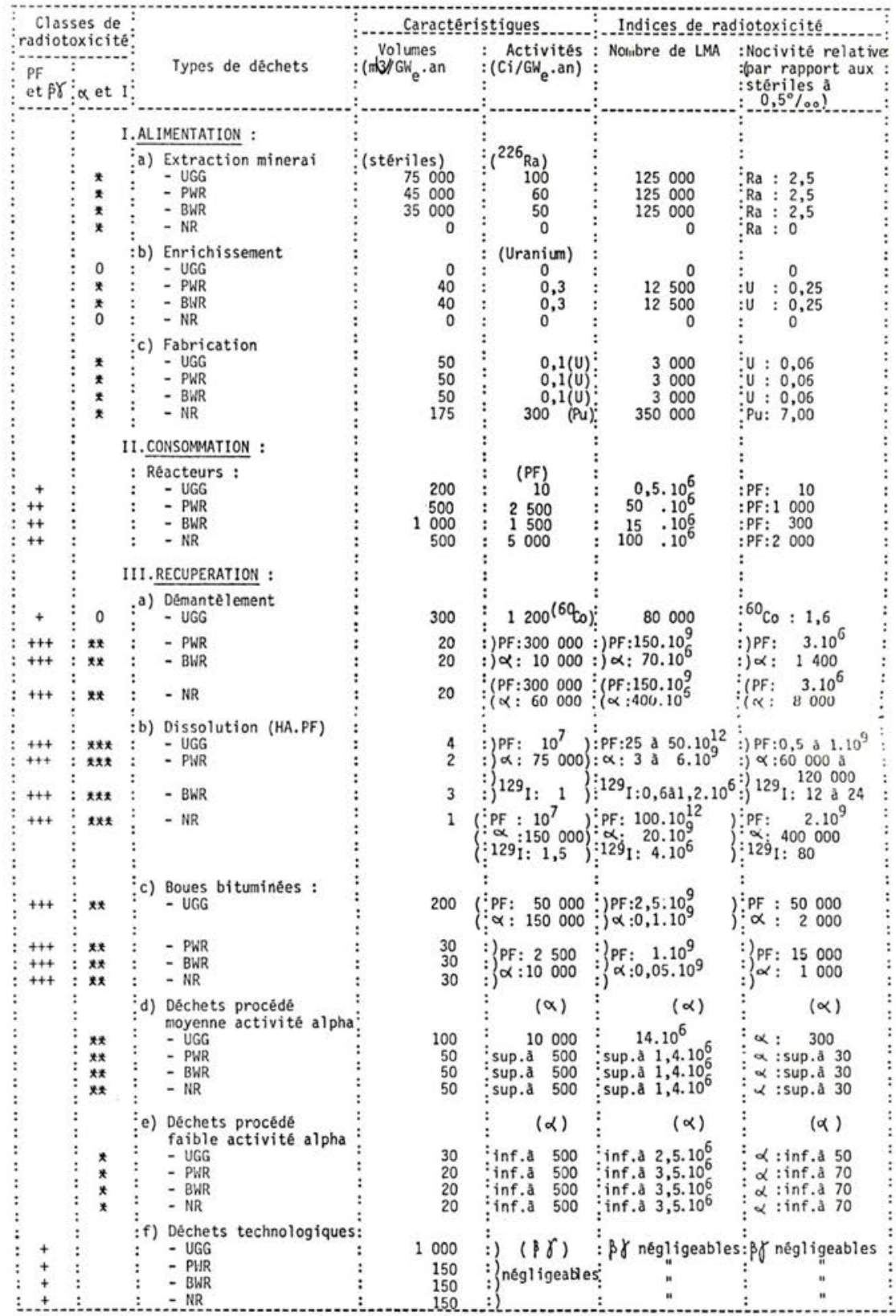

UGG : filière uranium-graphite-gaz. PWR : réacteur à eau sous pression. BWR : réacteur à eau bouillante. NR : filière à neutrons rapides. 
radiotoxicité et celui de nocivité relative. L'indice de radiotoxicité des déchets s'exprime ici par le nombre de LMA (Limite maximale admissible dans l'eau pour le public ou $1 / 10$ de la CMA) virtuellement contenu dans un volume donné de déchets. Cet indice de radiotoxicité est donc égal au rapport de l'activité spécifique du déchet par sa LMA. La nocivité relative est la comparaison de deux indices de radiotoxicité : celui du déchet et celui d'un produit

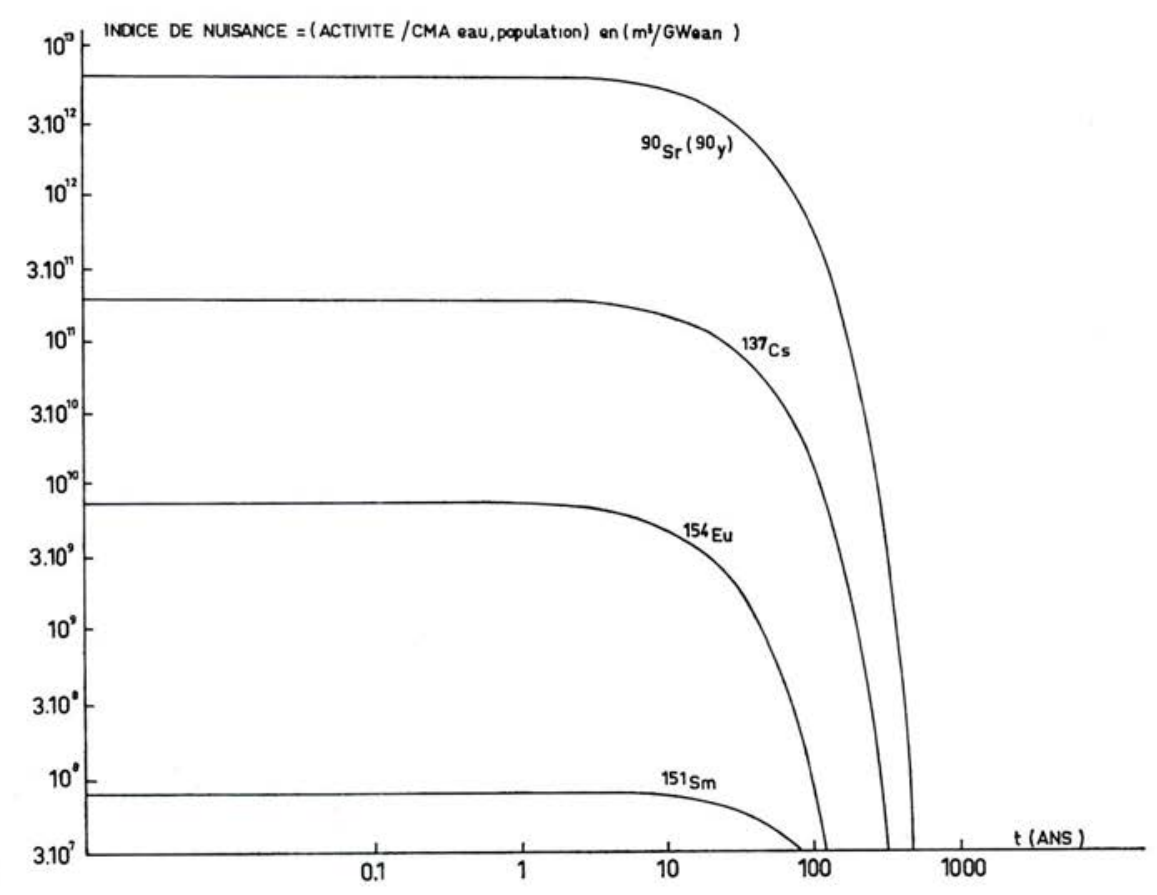

FIG. 12. - Déchets de haute activité d'un réacteur thermique $\left(1000 \mathrm{MW}_{e}\right)(33000 \mathrm{MWj} / \mathrm{t})$.

radioactif «naturel» pris pour référence (ici les «stériles» de minerais à $0,5 \%$ d'uranium). En général les nocivités et les volumes varient en sens inverse, ce qui est bien commode pour établir une stratégie du confinement final des déchets. Ce confinement a pour but d'isoler le déchet radioactif de son environnement jusqu'à ce que, par décroissance radioactive, le déchet soit devenu quasi-inactif. Un tel processus se déroule plus ou moins rapidement. En fait, on peut discerner dans l'évolution de la radioactivité artificielle engendrée par l'énergie nucléo-électrique au moins deux périodes :

- Une première période, qui concerne essentiellement les produits de fission et d'activation; de ceux-ci, après une dizaine d'années de décroissance, il ne reste plus guère que trois ou quatre isotopes assez radiotoxiques, et

vol. $14-\mathrm{N}^{\circ} 3$ 
passés 1000 ans, cette radiotoxicité (essentiellement bêta-gamma) a pratiquement disparu ( fig. 12). On pourrait appeler de tels radioéléments, par une analogie osée avec les produits biodégradables, des déchets radiodégradables.

- Une deuxième période, pratiquement illimitée, concerne essentiellement les transuraniens (presque tous émetteurs alpha) et l'iode 129 ( fig. 13).

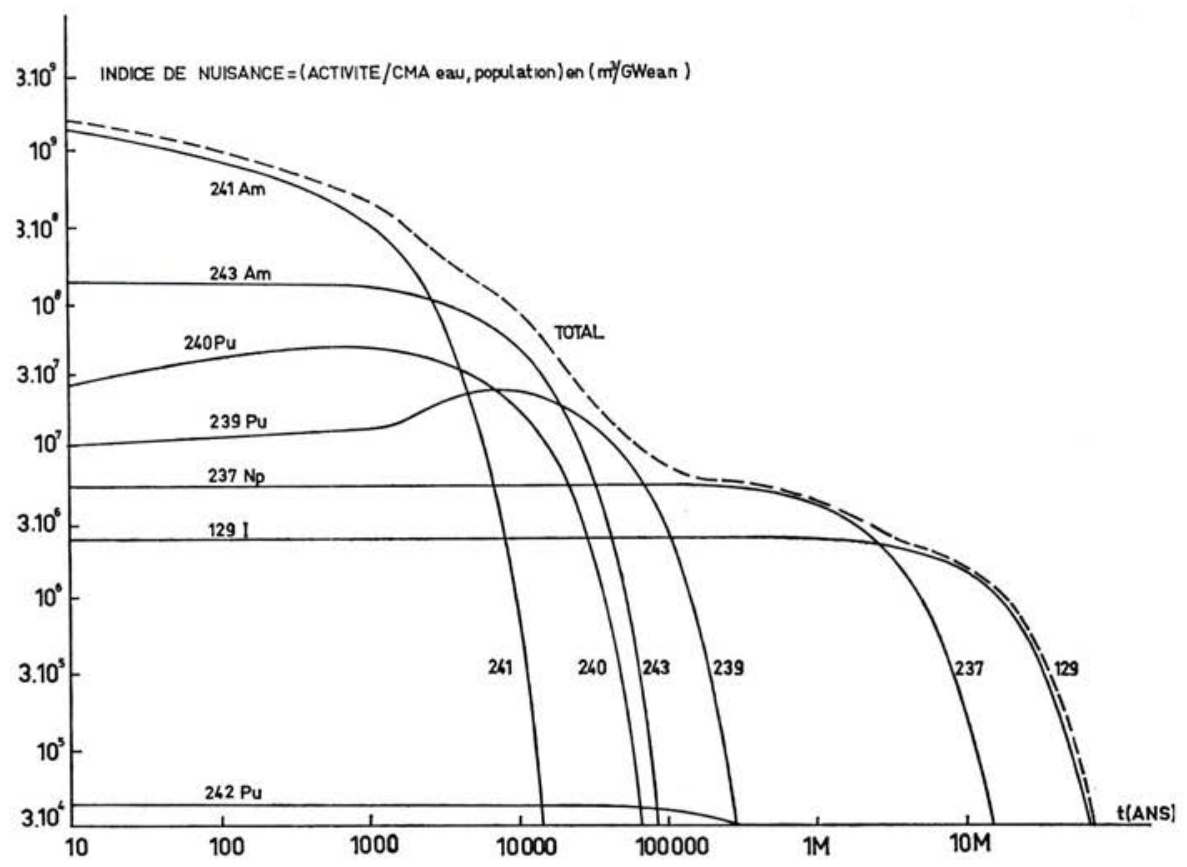

FIG. 13. - Déchets haute activité d'un réacteur thermique $\left(1000 \mathrm{MW}_{e}\right)(33000 \mathrm{MWj} / \mathrm{t})$ : — taux d'extraction U et Pu : $99,5 \%$ - taux d'extraction trans $\mathrm{U}: 0 \%$.

Quand à la radiotoxicité du radium et de ses descendants contenus dans les déchets, elle résulte, par filiation, de la décroissance de l'uranium naturel qui a alimenté les réacteurs et qui subsiste, après traitement du combustible, dans les déchets. Ce radium ainsi «reconstitué » dans les déchets de l'industrie nucléo-électrique ne constitue pas un risque supérieur à celui qu'il constituait en restant dans la nature. D'une façon plus générale, un chercheur américain, Clatborne, a établi que la nocivité des déchets produits par les réacteurs thermiques de l'industrie nucléo-électrique était inférieure à celle de l'uranium dont ils étaient issus. Cette constation n'a pas d'intérêt pratique immédiat car les concentrations de déchets ne sont pas les mêmes, ni situées au même endroit, que celles du minerai. Mais d'un point de vue philosophique, il est intéressant de constater qu'à très long terme l'industrie nucléaire diminue la nuisance radiologique du globe. 
En résumé, en supposant un développement maximal de l'énergie nucléoélectrique et avec une évaluation maximale du taux de production des déchets radioactifs, on aboutit à un inventa re (tableau II) qui constitue un plafond de la production des divers types de déchets. Ceux-ci ont été classés plus ou moins arbitrairement en fonction de leur radiotoxicité, et distingués en « radiodégradables » ou permanents.

Le but final étant d'isoler ces déchets qui peuvent engendrer des effluents " différés ", notamment par léchage au contact des eaux souterraines, on interpose des barrières entre eux et l'environnement. Ces barrières doivent résister jusqu'à élimination de la radioactivité, ou au moins de la radiotoxicité. On distingue ainsi :

- la première barrière : matrice dans laquelle est noyée la matière radioactive; cette matrice peut être en verre, en bitume, en résine, en béton, en métal, etc;

- la deuxième barrière enveloppe la précédente : acier, plomb, toute matière solide susceptible d'assurer une étanchéité au-delà de la première barrière et éventuellement de la protéger contre les chocs (notamment pendant les manipulations);

- la troisième barrière est de nature géochimique; c'est un obstacle qui agit à la façon d'un dispositif retardateur, en fixant les éléments radioactifs et en les empêchant de se disséminer dans le milieu;

- la quatrième barrière est la seule qui doive assurer un confinement à l'épreuve du temps quand les trois précédentes ont disparu (par corrosion, usure, etc.). Ce peuvent être des formations géologiques carcérales profondes (dômes de sel gemme, granit, etc.). Mais ce peuvent être aussi de simples " mausolées" construits en surface, de main d'homme, pour y entreposer les déchets " radiodégradables " jusqu'à leur élimination par disparition de leur radioactivité. Ce peut être encore l'envoi dans l'espace, sous de faibles volumes, il est vrai - ce qui conviendrait aux transuraniens si on les isolait des produits de fission.

Mais on peut également transformer un produit radioactif en un autre, de moindre longévité; c'est ce qu'on fait pour le plutonium lorsqu'on s'en sert comme combustible, notamment dans les réacteurs surrégénérateurs (Phénix, Superphénix, etc.). En recyclant le plutonium dans les réacteurs, on évite d'en faire un déchet. De la même façon, on peut envisager de transmuter les transuraniens, par exemple en les «brûlant » dans ces remarquables " incinérateurs " de déchets que seraient les réacteurs surrégénérateurs; il faudra, cependant, veiller à ce que les nouveaux radioisotopes ainsi obtenus par transmutation aient des vies radioactives suffisamment courtes pour ne pas poser de problèmes d'élimination plus sévères que ceux qu'on espérait résoudre.

Les solutions techniques envisageables ne manquent donc pas; leur étude est plus ou moins avancée; aini, la France est en tête du peloton mondial dans la technique de la vitrification des déchets. Les stratégies possibles de 
ÉVALUATION DE LA LIMITE SUPÉRIEURE DE L'ENCOMBREMENT DES DÉCHETS SOLIDES D'ORIGINE NUCLÉO-ÉLECTRIQUE FRANÇAISE (cumulés jusqu'au $\mathrm{XXI}^{\circ}$ siècle)

\begin{tabular}{|c|c|c|c|c|c|c|c|}
\hline \multirow{3}{*}{$\begin{array}{c}\text { Classe } \\
\text { de } \\
\text { nocivité }\end{array}$} & \multirow{3}{*}{$\begin{array}{l}\text { Radio- } \\
\text { toxicité }\end{array}$} & \multirow{2}{*}{\multicolumn{2}{|c|}{$\begin{array}{c}\text { Nocivité } \\
\text { relative }\left(^{*}\right) \\
\text { (ordre } \\
\text { de grandeur) }\end{array}$}} & \multicolumn{3}{|c|}{ Déchets (**) } & \multirow{3}{*}{ Observations } \\
\hline & & & & \multirow{2}{*}{ Origine } & \multirow{2}{*}{$\begin{array}{l}\text { Condi- } \\
\text { tionnement } \\
\text { (actuel) }\end{array}$} & \multirow{2}{*}{$\begin{array}{c}\text { Volume } \\
\text { cumulé } \\
\left(\mathrm{m}^{3}\right) \\
\text { (ordre } \\
\text { de grandeur) }\end{array}$} & \\
\hline & & $\mathbf{P}$ & $\mathrm{T}$ & & & & \\
\hline \multirow[t]{3}{*}{ H.A. } & $\begin{array}{l}\alpha \\
\text { I } \\
\text { PF }\end{array}$ & $\begin{array}{c}10^{5} \\
20 \\
- \\
\end{array}$ & $\begin{array}{c}- \\
10^{9}\end{array}$ & $\begin{array}{l}\text { Dissolution } \\
\text { des combustibles }\end{array}$ & Verre & 4000 & - \\
\hline & $\begin{array}{c}\alpha \\
\mathrm{PF}\end{array}$ & $\begin{array}{c}10^{3} \\
-\end{array}$ & $\overline{10^{6}}$ & $\begin{array}{l}\text { "Coques " de } \\
\text { dissolution des } \\
\text { combustibles } \\
\text { gainés }\end{array}$ & $\begin{array}{l}\text { non défini } \\
\text { (ou } \\
\text { ciment) }\end{array}$ & 40000 & - \\
\hline & $\begin{array}{c}\alpha \\
\text { PF }\end{array}$ & $10^{3}$ & $\begin{array}{c}- \\
10^{4} \\
\end{array}$ & $\begin{array}{l}\text { Boue de dis- } \\
\text { solution des } \\
\text { combustibles }\end{array}$ & Bitume & 60000 & $\begin{array}{l}\text { non condi- } \\
\text { tionné : } \\
200000 \mathrm{~m}^{3}\end{array}$ \\
\hline \multirow[t]{2}{*}{ M.A.P. } & $\alpha$ & $10^{2}$ & - & $\begin{array}{l}\text { Retraitement : } \\
\text { déchets de } \\
\text { procédé (M.A.) }\end{array}$ & Fûts $\|\alpha\|$ & 100000 & - \\
\hline & $\alpha$ & $<10^{2}$ & - & $\begin{array}{l}\text { Retraitement : } \\
\text { déchets de } \\
\text { procédé (F.A.) }\end{array}$ & $\begin{array}{l}\text { Fûts } \\
\text { non }((\alpha))\end{array}$ & 40000 & - \\
\hline M.A.T. & PF & - & $10^{-}$ & $\begin{array}{l}\text { Réacteurs (boues, } \\
\text { filtres, divers) }\end{array}$ & $\begin{array}{c}\text { Ciment ou } \\
\text { bitume }\end{array}$ & 1000000 & - \\
\hline \multirow[t]{5}{*}{ F.A. } & $\overline{{ }^{60} \mathrm{Co}}$ & - & 2 & $\begin{array}{c}\text { Chemises graph. } \\
\text { des réacteurs } \\
\text { graphite gaz }\end{array}$ & - & 20000 & $\begin{array}{l}\text { Pour } 60 \\
\mathrm{GW}_{e} \text {.an GGU } \\
\text { en l'an } 2000\end{array}$ \\
\hline & $\beta \gamma$ & - & $\varepsilon$ & $\begin{array}{l}\text { Retraitement : } \\
\text { déchets techno- } \\
\text { logiques }\end{array}$ & Fûts & 300000 & - \\
\hline & $\mathrm{Pu}$ & 0,1 & - & $\begin{array}{l}\text { Fabrication de } \\
\text { combustible } \\
\text { des filières : } \\
\text { neutrons rapides } \\
\text { autres }\end{array}$ & - & 20000 & $\begin{array}{c}\text { Pour } 100 \\
\mathrm{GW}_{e} \text {. an NR } \\
\text { en l'an } 2000 \\
-\end{array}$ \\
\hline & $\mathrm{U}$ & 0,2 & - & $\begin{array}{r}\text { Enrichissement } \\
\text { de l'uranium }\end{array}$ & - & 80000 & - \\
\hline & $\mathrm{Ra}$ & 2 & - & $\begin{array}{l}\text { Stériles } \\
\text { de minerais }\end{array}$ & - & 100000000 & - \\
\hline
\end{tabular}

$\left(^{*}\right)$ Après 1 an de décroissance radioactive. ( $\left(^{* *}\right)$ Correspondant à $2000 \mathrm{GW}_{e}$ an.

Légende : P, Permanent ( $>10^{5}$ ans) $\mathrm{T}$, Temporaire $\left(<10^{3}\right.$ ans; H.A., Haute activité; M.A., Moyenne activité; F.A., Faible activité. 


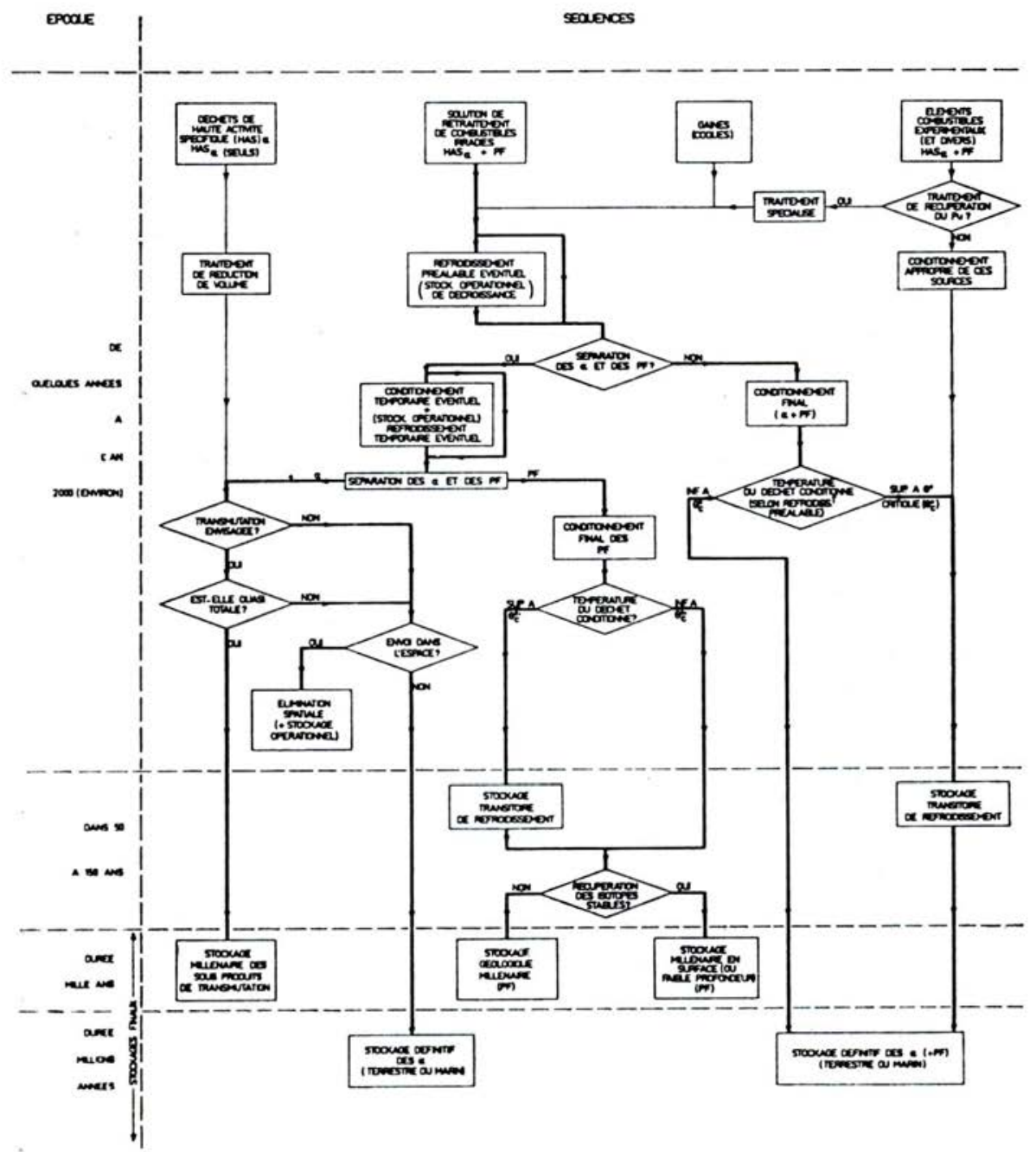

FIG. 14. - Options techniques à prendre pour les déchets de haute activité, d'après [6].

stockage des déchets ont fait l'objet d'une large concertation interministérielle; les options techniques apparaissent sous forme d'embranchements sur un ordinogramme (fig. 14). Il reste à faire des choix et c'est là le point le plus épineux. Mais il faut placer son espoir dans la sagesse, et tant pour la sûreté des installations nucléaires que pour celle du stockage final des déchets radioactifs, croire, comme TuBIANA, que le Refus du réel n'est qu'un épisode de la destinée humaine.

voL. $14-\mathrm{N}^{\circ} 3$ 


\section{BIBLIOGRAPHIE}

[1] Bourgeors J. Aspect technique de la sûreté des installations nucléaires. Ann. Mines 1974, janvier, 21-26.

[2] Sousselier Y. Les déchets radioactifs. Ann. Mines, 1974, janvier, 77-86.

[3] Servant J. La sécurité nucléaire en France. Ann. Mines, 1976, 182, n 3/4, 23-26.

[4] CoGNe F. La sûreté des réacteurs à eau sous pression, problèmes et études correspondantes. Ann. Mines, 1976, 182, $\mathrm{n}^{\circ}$ 3/4, 89-100.

[5] Justin F. La sûreté des réacteurs à neutrons rapides. Ann. Mines, 1976, 182, n 3/4, 117-122.

[6] Giraud B., Candes P. Les déchets radioactifs. Ann. Mines, 1976, 182, n 3/4, 145-162.

[7] Le nucléaire en questions. Rev. Gén. Nucl., 1977, $\mathrm{n}^{\circ}$ spécial.

[8] TanguY P. Que faut-il penser du rapport Rasmussen? Rev. Gén. Nucl., 1975, 1, $n^{\circ} 1,35-43$.

[9] Naudet R. Le phénomène d'OKLO, Rev. Gén. Nucl., 1975, 1, nº 1, 45-50.

[10] Gauvenet A. Les résidus radioactifs, problèmes actuels et perspectives. Rev. Gén. Nucl., 1975, 1, $\mathrm{n}^{\circ}$ 2, 113-118.

[11] Sousselier Y. Le transport des matières radioactives. Rev. Gén. Nucl., 1975, 1, n 5/6, 356-364.

[12] Tanguy P. La sûreté nucléaire et les méthodes probabilistes. Rev. Gén. Nucl., 1976, $\mathrm{n}^{\circ} 5,392-400$.

[13] CaRnino A. Application des méthodes probabilistes en matière de sûreté nucléaire. Rev. Gén. Nucl., 1976, $\mathrm{n}^{\circ}$ 5, 406-489.

[14] Gauvenet A., Giraud B. et al. Traitement et stockage des déchets radioactifs. Rev. Gén. Nucl., 1976, $\mathrm{n}^{\circ} 6$, 484-489.

[15] Livolant M., Barbreau A. et Costes D. Protection des centrales nucléaires contre les séismes. Rev. Gén. Nucl., 1977, n 1, 30-38.

[16] GeY A. La criticité : prévention, accidents et incidents, alarme, dosimétrie. Radioprotection, 1973, 8, $\mathrm{n}^{\circ} 2,97-117$.

[17] Gey A. Sûreté radiologique des installations et de leurs sites. Radioprotection, 1974, 9, $\mathrm{n}^{\circ} 3,233-243$.

[18] Évaluation des risques dus à un accident dans les installations nucléaires commerciales aux U.S.A., Rapport WASH-1400 de N. C. RaSmussen, condensé par A. GeY Radioprotection, 1974, 9, $\mathrm{n}^{\circ} \Delta, 325-332$.

[19] Clement B. Analyse de la sûreté des centrales électronucléaires françaises et critères. Radioprotection, 1975, 10, $\mathrm{n}^{\circ} 3,133-142$. 